\title{
Generalized Couch-Torrence symmetry for rotating extremal black holes in maximal supergravity
}

\author{
M. Cvetič, ${ }^{1,2}$ C. N. Pope, ${ }^{3,4}$ and A. Saha $\oplus^{3}$ \\ ${ }^{1}$ Department of Physics and Astronomy, University of Pennsylvania, Philadelphia, \\ Pennsylvania 19104, USA \\ ${ }^{2}$ Center for Applied Mathematics and Theoretical Physics, University of Maribor, \\ SI2000 Maribor, Slovenia \\ ${ }^{3}$ George P. \& Cynthia Woods Mitchell Institute for Fundamental Physics and Astronomy, \\ Texas A\&M University, College Station, Texas 77843, USA \\ ${ }^{4}$ DAMTP, Centre for Mathematical Sciences, Cambridge University, Wilberforce Road, \\ Cambridge CB3 OWA, United Kingdom
}

(Received 28 August 2020; accepted 11 September 2020; published 8 October 2020)

\begin{abstract}
The extremal Reissner-Nordström black hole admits a conformal inversion symmetry, in which the metric is mapped into itself under an inversion of the radial coordinate combined with a conformal rescaling. In the rotating generalization, Couch and Torrence showed that the Kerr-Newman metric no longer exhibits a conformal inversion symmetry, but the radial equation arising in the separation of the massless Klein-Gordon equation admits a mode-dependent inversion symmetry, where the radius of inversion depends upon the energy and azimuthal angular momentum of the mode. It was more recently shown that the static four-charge extremal black holes of STU supergravity (i.e., $\mathcal{N}=2$ supergravity in four dimension coupled to three vector multiplets) admit a generalization of the conformal inversion symmetry, in which the conformally inverted metric is a member of the same four-charge black hole family but with transformed charges. In this paper we study further generalizations of these inversion symmetries, within the general class of extremal STU supergravity black holes. For the rotating black holes, where again the massless Klein-Gordon equation is separable, we show that examples with four electric charges exhibit a generalization of the CouchTorrence symmetry of the radial equation. Now, as in the conformal inversion of the static specializations, the inversion of the radial equation maps it to the radial equation for a rotating black hole with transformed electric charges. We also study the inversion transformations for the general case of extremal Bogomol'nyi-Prasad-Sommerfield STU black holes carrying eight charges (four electric plus four magnetic), and argue that analogous generalizations of the inversion symmetries exist for both the static and the rotating cases.
\end{abstract}

DOI: 10.1103/PhysRevD.102.086007

\section{INTRODUCTION}

It was observed many years ago that the extremal limit of the Reissner-Nordström black hole exhibits a remarkable conformal inversion symmetry, in which an inversion of the radial coordinate, which maps the near-horizon region to the region near infinity, combined with a conformal rescaling, transforms the original metric back into itself [1]. Explicitly, consider the original extremal ReissnerNordström metric written in the isotropic form

Published by the American Physical Society under the terms of the Creative Commons Attribution 4.0 International license. Further distribution of this work must maintain attribution to the author(s) and the published article's title, journal citation, and DOI. Funded by SCOAP ${ }^{3}$. $d s^{2}=-\left(1+\frac{Q}{r}\right)^{-2} d t^{2}+\left(1+\frac{Q}{r}\right)^{2}\left(d r^{2}+r^{2} d \Omega_{2}^{2}\right)$,

where $d \Omega_{2}^{2}$ is the metric on the unit 2-sphere. The horizon is located at $r=0$. Performing the inversion to a new radial coordinate

$$
\tilde{r}=\frac{Q^{2}}{r}
$$

one finds that the conformally related metric $d \tilde{s}^{2}$ defined by

$$
d s^{2}=\frac{Q^{2}}{\tilde{r}^{2}} d \tilde{s}^{2}
$$


is given by

$$
d \tilde{s}^{2}=-\left(1+\frac{Q}{\tilde{r}}\right)^{-2} d t^{2}+\left(1+\frac{Q}{\tilde{r}}\right)^{2}\left(d \tilde{r}^{2}+\tilde{r}^{2} d \Omega_{2}^{2}\right) .
$$

Thus after the conformal inversion, the resulting metric $d \tilde{s}^{2}$ is again the extremal Reissner-Nordström metric and takes the identical form to the original metric (1.1) [1]. The radius of the inversion in (1.2) is equal to the electric charge $Q$ (and hence also the mass).

The conformal inversion symmetry of the extremal Reissner-Nordström metric has been employed in a number of papers (see, for example, [2-4]) in order to relate some of the recent observations about the asymptotic behavior of Klein-Gordon and other fields on the future horizon of an extremal black hole (see, for example, [5,6]) to the asymptotic behavior of these fields at future null infinity.

It was shown also in [1] that the conformal inversion symmetry of the extremal Reissner-Nordström metric does not generalize to the rotating case, namely the extremal Kerr-Newman metric. It was, however, observed that if one considers a massless Klein-Gordon field $\Phi$ in the extremal Kerr-Newman background, then after performing a separation of variables the differential equation for the radial function exhibits a remarkable inversion symmetry. This involves a radius of inversion that depends not only on the black hole charge and rotation parameters but also on the separation constants $\omega$ and $m$ that arise in the factorized solutions

$$
\Phi(t, r, \theta, \varphi)=R(r) S(\theta) e^{-\mathrm{i} \omega t} e^{\mathrm{i} m \varphi}
$$

Thus the inversion radius is different for different modes.

In [4], conformal inversions of a class of more general static extremal black holes were investigated. Specifically, the static extremal four-charge black hole solutions [7] of the four-dimensional STU supergravity (i.e., $\mathcal{N}=2$ supergravity in four dimension coupled to three vector multiplets) theory were studied. ${ }^{1}$ Thus there are four electromagnetic field strengths in total, each of which can carry, in general, independent electric and magnetic charges. The general eight-charge solution is quite complicated but the special case where each field strength carries just an electric charge is much simpler, with the metric being given by

$$
d s^{2}=-H^{-1 / 2} d t^{2}+H^{1 / 2}\left(d r^{2}+r^{2} d \Omega_{2}^{2}\right),
$$

\footnotetext{
${ }^{1}$ This is also a consistent truncation of ungauged supergravity theory with maximal (both $\mathcal{N}=4$ and $\mathcal{N}=8$ ) supersymmetry. Black hole solutions of STU supergravity are generating solutions of the full maximally supersymmetric ungauged supergravity theories.
}

$$
H=\prod_{i=1}^{4}\left(1+\frac{Q_{i}}{r}\right),
$$

As was shown in [4], under the inversion

$$
\tilde{r}=\frac{\Pi^{2}}{r}, \quad \Pi \equiv \prod_{i=1}^{4} Q_{i}^{1 / 4},
$$

one finds that the conformally related metric $d \tilde{s}^{2}$ given by

$$
d s^{2}=\frac{\Pi^{2}}{\tilde{r}^{2}} d \tilde{s}^{2}
$$

takes the form

$$
\begin{gathered}
d \tilde{s}^{2}=-\tilde{H}^{-1 / 2} d t^{2}+\tilde{H}^{1 / 2}\left(d \tilde{r}^{2}+\tilde{r}^{2} d \Omega_{2}^{2}\right), \\
\tilde{H}=\prod_{i=1}^{4}\left(1+\frac{\tilde{Q}_{i}}{\tilde{r}}\right),
\end{gathered}
$$

where

$$
\tilde{Q}_{i}=\frac{\Pi^{2}}{Q_{i}}
$$

Thus the metric $d \tilde{s}^{2}$ obtained by the conformal inversion is in the same class of four-charge static extremal black holes as the original metric (1.6), but for new charges $\tilde{Q}_{i}$ related to the original charges $Q_{i}$ by (1.12) [4].

In the special case where all four charges are equal, the metric (1.6) reduces to the extremal Reissner-Nordström metric (1.1) and the conformal inversion gives back this metric again. A more general specialization where the conformal inversion becomes an actual symmetry is if the charges are set equal in pairs; for example,

$Q_{3}=Q_{1}, \quad Q_{4}=Q_{2}, \Rightarrow \tilde{Q}_{3}=\tilde{Q}_{1}=Q_{2}$,

$\tilde{Q}_{4}=\tilde{Q}_{2}=Q_{1}$,

and hence $\tilde{H}=H$ [4].

One purpose of the present paper is to investigate whether the observation of Couch and Torrence that the conformal inversion symmetry of the extremal static Reissner-Nordström metric has a corresponding inversion transformation of the radial equation in the rotating case might generalize to rotating versions of the four-charge extremal black holes in STU supergravity. We show that this question can be answered in the affirmative. Namely, we show that in the rotating extremal four-charge STU supergravity background, the radial equation for the separated massless Klein-Gordon equation indeed exhibits an inversion symmetry. As in the Kerr-Newman case, the inversion radius depends not only on the black hole charge 
and rotation parameters but also on the mode numbers $\omega$ and $m$ arising in the separation of variables. A new feature that we find for the general four-charge black holes is that after the inversion, the radial equation is not the same as the original radial equation but, rather, it is the radial equation for a transformed set of charge parameters. This is the analogue, for the rotating case, of the mapping of the charges (1.12) that was found for the static four-charge black holes.

Another purpose of this paper is to investigate conformal inversion in the most general setting of the eight-charge static extremal STU supergravity black holes and the analogous inversion of the Klein-Gordon radial equation in the extremal eight-charge rotating backgrounds. The goal is to establish whether these inversion transformations again provide a mapping back into the solution space of the eight-charge black holes.

It should be emphasized at this point that there exist disjoint classes of static extremal black holes in STU supergravity. The examples with four electric charges that we discussed above are contained within the class of BPS (Bogomol'nyi-Prasad-Sommerfield) extremal black holes. These are typically supersymmetric, preserving some fraction of the supersymmetry that is governed by the number, and the pattern, of the nonvanishing charges. These BPS black holes form the focus of our studies in this paper. Our findings, which we describe in detail later, suggest that the entire eight-charge static BPS family of black holes maps into itself under conformal inversion. We reach this conclusion by constructing explicitly the general eight-charge static BPS extremal family of black holes and studying the conditions that arise from requiring that the family map into itself under conformal inversion. Unlike the situation for the case of four electric charges which was studied in [4], it does not seem to be possible in the general case to give an elegant formula for the mapping of the charges under conformal inversion, analogous to that in Eq. (1.12). This is a consequence of the fact that the system of conditions resulting from the requirement of the existence of a conformal inversion mapping is underdetermined. That is to say, there are now fewer conditions than the number of unknowns (the eight mapped charges), and so there is not a unique solution. We have checked in many examples, and it appears that a mapping of charges always exists.

We also study the general eight-charge rotating extremal black holes. As in the four-charge specialization described earlier, here too the massless Klein-Gordon equation can be separated and the behavior of the radial wave equation under inversion can be investigated. We find that, as in the eight-charge static BPS extremal black holes described above, although we can write down the conditions for the inversion of the radial coordinate to give rise again to a radial equation for a set of mapped charges, it does not appear to be possible to give an elegant formula for the mapped charges. Again, the reason is that the system of conditions for invertability does not fully constrain the mapped charges. As in the static case, we may nevertheless argue that solutions for the mapped charges will exist.

There also exists a class of extremal black holes in STU supergravity that does not obey the BPS conditions. A simple example that was investigated in [4] was the static extremal Kaluza-Klein dyonic black hole, which in the language of STU supergravity corresponds to the case where just one of the four electromagnetic fields is turned on and carries independent electric and magnetic charges, with the other three electromagnetic fields being zero. It was shown in [4] that this metric does not map into any metric within the same family, under conformal inversion. This does not, however, necessarily provide a counterexample to the idea that the entire eight-charge family of nonBPS extremal black holes might map into itself under conformal inversion, since the conformal inversion of the Kaluza-Klein dyon might in principle be an eight-charge non-BPS extremal solution that did not lie within the original Kaluza-Klein dyonic subset. However, owing to the greater complexity of the general eight-charge non-BPS extremal solutions, we shall not pursue this question further in the present paper.

\section{INVERSION SYMMETRY OF RADIAL EQUATION FOR FOUR-CHARGE ROTATING BLACK HOLES}

Couch and Torrence observed that although there is no conformal inversion symmetry of the extremal KerrNewman metric (unlike the situation for the extremal Reissner-Nordström solution), there is nevertheless an inversion symmetry of the radial equation after one separates variables in the massless scalar wave equation in the Kerr-Newman geometry [1]. Here, we show that there exists a generalization of this inversion symmetry for the radial equation arising from the separation of variables for the massless scalar wave equation in the four-dimensional four-charge rotating black hole solutions. The situation for the general four-charge case is reminiscent of the situation found in [4] for the entire metric of the four-charge extremal static black holes, namely, that the inversion applied to a generic four-charge case maps it into another case with different values of the four charges. In the special case of pairwise-equal charges, the inversion maps the radial equation into exactly the same radial equation. A special case of this, when all four charges are equal, reduces to the Kerr-Newman result found by Couch and Torrence.

A convenient presentation of the rotating black holes in four-dimensional STU supergravity carrying four independent charges can be found in $[8,9]$. The metric can be written as [9]: 


$$
\begin{aligned}
d s^{2} & =-\frac{\rho^{2}-2 m r}{W}\left(d t+\mathcal{B}_{(1)}\right)^{2}+W\left(\frac{d r^{2}}{\Delta}+d \theta^{2}+\frac{\Delta \sin ^{2} \theta d \tilde{\phi}^{2}}{\rho^{2}-2 m r}\right), \\
\mathcal{B}_{(1)} & =\frac{2 m a \sin ^{2} \theta\left(r \Pi_{c}-(r-2 m) \Pi_{s}\right)}{\rho^{2}-2 m r} d \tilde{\phi}, \quad \rho^{2}=r^{2}+a^{2} \cos ^{2} \theta, \quad \Delta=r^{2}-2 m r+a^{2}, \\
W^{2} & =R_{1} R_{2} R_{3} R_{4}+a^{4} \cos ^{4} \theta+\left[2 r^{2}+2 m r \sum_{i} s_{i}^{2}+8 m^{2}\left(\Pi_{c}-\Pi_{s}\right) \Pi_{s}-4 m^{2} \sum_{i<j<k} s_{i}^{2} s_{j}^{2} s_{k}^{2}\right] a^{2} \cos ^{2} \theta, \\
R_{i} & =r+2 m s_{i}^{2}, \quad \Pi_{c}=\prod_{i} c_{i}, \quad \Pi_{s}=\prod_{i} s_{i} .
\end{aligned}
$$

The four physical charges are given by $q_{i}=2 m s_{i} c_{i}=m \sinh 2 \delta_{i}$. Extremality is achieved by taking $m=a$, and the horizon is then at $r=a$. It is straightforward to separate variables in the massless wave equation $\square \Psi=0$ in this background. ${ }^{2}$ Writing

$$
\Psi=e^{-\mathrm{i} \omega t+\mathrm{i} m \tilde{\phi}} R(\hat{r}) S(y),
$$

where we write $y=\cos \theta$ and define

$$
\hat{r}=r-a
$$

(so that the horizon is located at $\hat{r}=0$ ), we find that $R$ and $S$ satisfy the equations

$$
\hat{r}^{2} R^{\prime \prime}+2 \hat{r} R^{\prime}+(H-\lambda) R=0, \quad\left(1-y^{2}\right) S^{\prime \prime}-2 y S^{\prime}-\left(\frac{m^{2}}{1-y^{2}}+a^{2} \omega^{2}\left(1-y^{2}\right)-\lambda\right) S=0,
$$

where $\lambda$ is the separation constant and

$$
\begin{aligned}
H= & \omega^{2} \hat{r}^{2}+2 a \omega^{2}\left(2+\sum_{i} s_{i}^{2}\right) \hat{r}+2 a^{2} \omega^{2}\left(4+3 \sum_{i} s_{i}^{2}+2 \sum_{i<j} s_{i}^{2} s_{j}^{2}\right) \\
& +\left[8 a^{3} \omega^{2}\left(1+\sum_{i} s_{i}^{2}+\sum_{i<j} s_{i}^{2} s_{j}^{2}+\sum_{i<j<k} s_{i}^{2} s_{j}^{2} s_{k}^{2}\right)-4 a^{2} \omega m\left(\Pi_{c}-\Pi_{s}\right)\right] \frac{1}{\hat{r}} \\
& +\left[a^{2} m^{2}-4 a^{3} \omega m\left(\Pi_{c}+\Pi_{s}\right)+4 a^{4} \omega^{2}\left(1+\sum_{i} s_{i}^{2}+\sum_{i<j} s_{i}^{2} s_{j}^{2}+\sum_{i<j<k} s_{i}^{2} s_{j}^{2} s_{k}^{2}+2 \Pi_{s}\left(\Pi_{c}+\Pi_{s}\right)\right)\right] \frac{1}{\hat{r}^{2}} .
\end{aligned}
$$

A straightforward calculation then shows that writing $H$ as $H\left(\hat{r}, \delta_{i}\right)$, with $s_{i}=\sinh \delta_{i}$, etc., then

$$
H\left(\hat{r}, \delta_{i}\right)=H\left(\frac{\beta^{2}}{\hat{r}}, \tilde{\delta}_{i}\right)
$$

where

$$
\beta^{2}=-\frac{a m}{\omega}+2 a^{2}\left(\Pi_{c}+\Pi_{s}\right)
$$

and the redefined charge parameters $\tilde{\delta}_{i}$ are related to the original parameters $\delta_{i}$ by the involution $\tilde{\delta}_{i}=\frac{1}{2}\left(\delta_{1}+\delta_{2}+\delta_{3}+\delta_{4}\right)-\delta_{i}$, i.e.,

\footnotetext{
${ }^{2}$ The massless wave equation is separable also in the nonextremal case, $m \neq a$, as shown in [10].
} 


$$
\begin{array}{ll}
\tilde{\delta}_{1}=\frac{1}{2}\left(\delta_{2}+\delta_{3}+\delta_{4}-\delta_{1}\right), & \tilde{\delta}_{2}=\frac{1}{2}\left(\delta_{1}+\delta_{3}+\delta_{4}-\delta_{2}\right), \\
\tilde{\delta}_{3}=\frac{1}{2}\left(\delta_{1}+\delta_{2}+\delta_{4}-\delta_{3}\right), & \tilde{\delta}_{4}=\frac{1}{2}\left(\delta_{1}+\delta_{2}+\delta_{3}-\delta_{4}\right) .
\end{array}
$$

Let us now define a (dimensionless) coordinate $x=\beta / \hat{r}$. Viewing the radial function $R$ as now being a function $P(x)$, we see that it satisfies

$$
x^{2} \partial_{x}^{2} P(x)+f\left(x, \delta_{i}\right) P(x)=0,
$$

where

$$
\begin{aligned}
f\left(x, \delta_{i}\right) & =\frac{1}{x^{2}}\left[H\left(\frac{\beta}{x}, \delta_{i}\right)-\lambda\right] \\
& =\beta^{2} \omega^{2}+\frac{\beta^{2} \omega^{2}}{x^{4}}+\frac{C_{+}\left(\delta_{i}\right)}{x}+\frac{C_{-}\left(\delta_{i}\right)}{x^{3}}+\frac{C_{0}\left(\delta_{i}\right)-\lambda}{x^{2}},
\end{aligned}
$$

with

$$
\begin{aligned}
C_{+}\left(\delta_{i}\right) & =4 a \beta \omega^{2}\left(\Pi_{c}-\Pi_{s}\right), \quad C_{-}\left(\delta_{i}\right)=a \beta \omega^{2} \sum_{i} \cosh 2 \delta_{i}, \\
C_{0}\left(\delta_{i}\right) & =a^{2} \omega^{2}\left[2+\frac{1}{2} \sum_{i<j}\left(\cosh 2\left(\delta_{i}-\delta_{j}\right)+\cosh 2\left(\delta_{i}+\delta_{j}\right)\right)\right] .
\end{aligned}
$$

One can easily verify that $\beta$ and $C_{0}$ are invariant under $\delta_{i} \rightarrow \tilde{\delta}_{i}$, while

$$
C_{+}\left(\delta_{i}\right)=C_{-}\left(\tilde{\delta}_{i}\right), \quad C_{-}\left(\delta_{i}\right)=C_{+}\left(\tilde{\delta}_{i}\right) .
$$

It follows that

$$
f\left(\frac{1}{x}, \delta_{i}\right)=x^{4} f\left(x, \tilde{\delta}_{i}\right)
$$

and so if $P\left(x, \delta_{i}\right)$ is a solution of (2.9) then defining

$$
\tilde{x}=\frac{1}{x}, \quad \tilde{P}(\tilde{x})=\frac{1}{x} P(x),
$$

the function $\tilde{P}(\tilde{x})$ will solve the tilded equation

$$
\tilde{x}^{2} \partial_{\tilde{x}}^{2} \tilde{P}(\tilde{x})+f\left(\tilde{x}, \tilde{\delta}_{i}\right) \tilde{P}(\tilde{x})=0 .
$$

Thus a solution $P(x)$ of the radial equation with charge parameters $\delta_{i}$ maps into a solution of the inverted radial equation with charge parameters $\tilde{\delta}_{i}$ given by (2.8).

Note that in the specialization to pairwise-equal charges, such as $\delta_{3}=\delta_{1}$ and $\delta_{4}=\delta_{2}$, one has

$$
\tilde{\delta}_{1}=\tilde{\delta}_{3}=\delta_{2}, \quad \tilde{\delta}_{2}=\tilde{\delta}_{4}=\delta_{1},
$$

and so in this case $H\left(x, \delta_{i}\right)=H\left(\frac{1}{x}, \delta_{i}\right)$ (since the function $H$ is symmetrical in the charge parameters). The constant $\beta$ in this pairwise-equal case is given by

$\beta^{2}=-\frac{a m}{\omega}+2 a^{2}\left(\cosh ^{2} \delta_{1} \cosh ^{2} \delta_{2}+\sinh ^{2} \delta_{1} \sinh ^{2} \delta_{2}\right)$.

Thus in this case, and in its further specialization to $\delta_{1}=\delta_{2}$ (all charges equal, i.e., the Kerr-Newman solution studied by Couch and Torrence), the inversion is an actual symmetry of the radial equation.

\section{A. Conformal inversion in the static limit}

It is interesting to look at the limit where the extremal four-charge metric (2.1) (with $m=a$ ) reduces to the metric of extremal four-charge static black holes, first obtained as a BPS black hole solution in [7]. Since the physical charges in the extremal rotating metric are given by $q_{i}=a \sinh 2 \delta_{i}$, one must send the charge parameters $\delta_{i}$ to infinity at the same time as sending the rotation parameter $a$ to zero, so as to hold the $q_{i}$ finite, and so one has

$$
q_{i}=\frac{a}{2} e^{2 \delta_{i}}
$$

fixed, and the metric becomes

$d s^{2}=-\left(\prod_{i} H_{i}\right)^{-\frac{1}{2}} d t^{2}+\left(\prod_{i} H_{i}\right)^{\frac{1}{2}}\left(d r^{2}+r^{2} d \Omega^{2}\right)$

in the extremal static limit, where $H_{i}=1+q_{i} r^{-1}$. After the inversion, and using (2.8), one has

$$
\tilde{q}_{i}=\frac{a}{2} e^{2 \tilde{\delta}_{i}}=\frac{a}{2} e^{\delta_{1}+\delta_{2}+\delta_{3}+\delta_{4}-2 \delta_{i}},
$$

and hence

$$
\tilde{q}_{i}=\frac{Q^{2}}{q_{i}}, \quad Q^{4} \equiv \prod_{i} q_{i} .
$$

This is precisely the relation between untilded and tilded charges that was found in [4] (and which we summarized in the Introduction) for the four-charge static metrics and the conformally related inverted metrics. In that case, the transformation mapped the entire metric into another metric within the same four-charge class. Thus the inversion symmetry (up to charge transformations) of the radial equation in the rotating case, which we exhibited above, becomes an inversion symmetry (up to charge transformations) of the entire metric in the static limit. 


\section{INVERSION SYMMETRY OF RADIAL EQUATION FOR PAIRWISE EQUAL DYONIC STU BLACK HOLES}

The generalization of the Couch-Torrence inversion symmetry of the separated radial equation to the general case of the eight-charge dyonic rotating extremal black holes of STU supergravity is rather complicated, and we shall not present it here. It becomes much more manageable in special cases, such as the case with four electric charges, which we discussed previously. Another case that is relatively straightforward is when the field strengths of STU supergravity are set equal in pairs, with each of the two remaining independent fields carrying independent electric and magnetic charges.

From the paper [11] of Chow and Compère, after separating variables in the massless Klein-Gordon equation for the eight-charge rotating STU black holes, the radial equation takes the form

$$
\begin{aligned}
& \partial_{r}\left(R \partial_{r} P(r)\right)+H P(r)=0, \\
& H=\frac{\omega^{2} W_{r}^{2}-2 a \omega k L_{r}+a^{2} k^{2}}{R}+\lambda,
\end{aligned}
$$

where $R=r^{2}-2 m r+a^{2}-n^{2}$, the separation constant is $\lambda$, and the factorized solutions are taken to have the form $\Psi=e^{-\mathrm{i} \omega t+\mathrm{i} k \varphi} P(r) S(\theta)$. (We follow [11] and use $k$ rather than $m$ for the azimuthal quantum number, since $m$ is used here to denote the black-hole mass parameter.) The constant $n$ is given by

$$
n=-m \frac{\nu_{1}}{\nu_{2}}
$$

(this is the condition for the physical NUT (Newman-UntiTamborino) charge $N=m \nu_{1}+n \nu_{2}$ to be zero), and the functions $W_{r}$ and $L_{r}$ in the extremal case are given by

$$
\begin{aligned}
W_{r}^{2} & =\rho^{4}+4 M \rho^{2}(\rho+m)+L_{r}^{2}, \\
L_{r} & =2 a^{2}\left(\frac{\nu_{2}}{m} \rho+\left(\nu_{2}+2 D\right)\right),
\end{aligned}
$$

where we have defined

$$
\rho=r-m,
$$

and the quantities $\nu_{1}, \nu_{2}$, and $D$ are given, in the pairwiseequal case, by

$$
\begin{aligned}
\nu_{1}= & -\frac{1}{2} \sinh 2 \delta_{1} \sinh 2 \gamma_{1}-\frac{1}{2} \sinh 2 \delta_{2} \sinh 2 \gamma_{2}, \\
\nu_{2}= & \frac{1}{2} \cosh 2 \delta_{1} \cosh 2 \gamma_{2}+\frac{1}{2} \cosh 2 \delta_{2} \cosh 2 \gamma_{1}, \\
D= & \frac{1}{4}\left(\cosh 2 \delta_{1} \cosh 2 \gamma_{2}-1\right)\left(\cosh 2 \delta_{2} \cosh 2 \gamma_{1}-1\right) \\
& +\frac{1}{4} \sinh 2 \delta_{1} \sinh 2 \delta_{2} \sinh 2 \gamma_{1} \sinh 2 \gamma_{2} .
\end{aligned}
$$

The physical mass $M$, given in general by $M=m \mu_{1}+n \mu_{2}$ [11], is given in the pairwise-equal case by $M=m \nu_{2}-n \nu_{1}$ (since then $\mu_{1}=\nu_{2}$ and $\mu_{2}=-\nu_{1}$ ). The four physical electric and magnetic charges $\left(Q_{1}, Q_{2}, P_{1}, P_{2}\right)$ carried by the two independent field strengths $F_{1}$ and $F_{2}$ are given in terms of the boost parameters $\left(\delta_{1}, \delta_{2}, \gamma_{1}, \gamma_{2}\right)$ by [11]

$Q_{1}=\frac{1}{2} m \sinh 2 \delta_{1} \cosh 2 \gamma_{2}+\frac{1}{2} n \cosh 2 \delta_{1} \sinh 2 \gamma_{1}$,

$Q_{2}=\frac{1}{2} m \sinh 2 \delta_{2} \cosh 2 \gamma_{1}+\frac{1}{2} n \cosh 2 \delta_{2} \sinh 2 \gamma_{2}$,

$P_{1}=\frac{1}{2} m \sinh 2 \gamma_{1} \cosh 2 \delta_{1}-\frac{1}{2} n \cosh 2 \gamma_{2} \sinh 2 \delta_{1}$,

$P_{2}=\frac{1}{2} m \sinh 2 \gamma_{2} \cosh 2 \delta_{2}-\frac{1}{2} n \cosh 2 \gamma_{1} \sinh 2 \delta_{2}$.

Viewing $H$, defined in (3.1), as a function of $\rho$, we find

$$
\begin{aligned}
H(\rho)= & \omega^{2}\left[\rho^{2}+\frac{\beta^{4}}{\rho^{2}}\right]+\frac{4 \nu_{2} a^{2} \omega^{2}}{m}\left[\rho+\frac{\beta^{2}}{\rho}\right] \\
& +4 \nu_{2} a^{2} \omega^{2}\left(1+\frac{a^{2} \nu_{2}}{m^{2}}\right)+\lambda,
\end{aligned}
$$

where

$$
\begin{aligned}
\beta^{2}= & 2 a^{2}\left(\nu_{2}+2 D\right)-\frac{a k}{\omega}, \\
= & a^{2}\left(1+\cosh 2 \delta_{1} \cosh 2 \delta_{2} \cosh 2 \gamma_{1} \cosh 2 \gamma_{2}\right. \\
& \left.+\sinh 2 \delta_{1} \sinh 2 \delta_{2} \sinh 2 \gamma_{1} \sinh 2 \gamma_{2}\right)-\frac{a k}{\omega} .
\end{aligned}
$$

Thus the function $H$ has the inversion symmetry

$$
H(\rho)=H\left(\frac{\beta^{2}}{\rho}\right) .
$$

This implies an inversion symmetry of the radial equation, namely, that if we define $\tilde{\rho}=\beta^{2} \rho^{-1}$, then the original radial equation (3.1) implies the inverted equation

$$
\partial_{\tilde{\rho}}\left(\tilde{\rho}^{2} \partial_{\tilde{\rho}} \tilde{P}(\tilde{\rho})\right)+H(\tilde{\rho}) \tilde{P}(\tilde{\rho})=0
$$

where

$$
\tilde{P}(\tilde{\rho})=\frac{\rho}{\beta} P(\rho)=\frac{\beta}{\tilde{\rho}} P\left(\frac{\beta}{\tilde{\rho}}\right) .
$$

Note that as in the case of the pairwise-equal specialization of the four electric charge black holes discussed previously, the inversion is an actual symmetry in this pairwise-equal dyonic charge case. One can easily verify that if the magnetic charges are set to zero, the inversion here reduces to the previous pairwise-equal result, with the radius of inversion $\beta$ reducing from (3.8) to (2.17). 


\section{A. Conformal inversion symmetry for static pairwise-equal dyonic black holes}

The static limit of the extremal rotating dyonic black holes with pairwise-equal charges is achieved by sending $m$ (and hence $n$ and $a$ ) to zero while sending the boost parameters to infinity, so as to keep the physical charges in (3.6) finite and nonzero. This can be done by sending

$m \rightarrow 4 \bar{m} e^{-4 \lambda}, \quad \delta_{i} \rightarrow \delta_{i}+\lambda, \quad \gamma_{i} \rightarrow \gamma_{i}+\lambda$,

and taking the limit $\lambda \rightarrow \infty$. The metric given in [11] becomes static, with

$$
\begin{aligned}
d s^{2}= & -\frac{r^{2}}{W} d t^{2}+\frac{W}{r^{2}}\left(d r^{2}+r^{2} d \Omega_{2}\right), \\
W= & r^{2}+2 M r+2\left(\bar{m}^{2}+\bar{n}^{2}\right) e^{2\left(\delta_{1}+\delta_{2}+\gamma_{1}+\gamma_{2}\right)}, \\
\bar{n}= & \bar{m} \frac{\cosh \left(\delta_{1}-\delta_{2}+\gamma_{1}-\gamma_{2}\right)}{\cosh \left(\delta_{1}-\delta_{2}-\gamma_{1}+\gamma_{2}\right)}, \\
M= & \bar{m} e^{\delta_{1}+\delta_{2}+\gamma_{1}+\gamma_{2}} \\
& \times \frac{\cosh ^{2}\left(\delta_{1}-\delta_{2}+\gamma_{1}-\gamma_{2}\right)+\cosh ^{2}\left(\delta_{1}-\delta_{2}-\gamma_{1}+\gamma_{2}\right)}{\cosh \left(\delta_{1}-\delta_{2}-\gamma_{1}+\gamma_{2}\right)} .
\end{aligned}
$$

The electric and magnetic charges in the static limit are given by ${ }^{3}$

$$
\begin{aligned}
Q_{1} & =\frac{\bar{m} e^{2 \delta_{1}}\left[e^{2 \delta_{1}}\left(e^{4 \gamma_{1}}+e^{4 \gamma_{2}}\right)+2 e^{2 \delta_{2}+2 \gamma_{1}+2 \gamma_{2}}\right]}{2\left(e^{2 \delta_{1}+2 \gamma_{2}}+e^{2 \delta_{2}+2 \gamma_{1}}\right)}, \\
Q_{2} & =\frac{\bar{m} e^{2 \delta_{2}}\left[e^{2 \delta_{2}}\left(e^{4 \gamma_{1}}+e^{4 \gamma_{2}}\right)+2 e^{2 \delta_{1}+2 \gamma_{1}+2 \gamma_{2}}\right]}{2\left(e^{2 \delta_{1}+2 \gamma_{2}}+e^{2 \delta_{2}+2 \gamma_{1}}\right)}, \\
P_{1} & =\frac{\bar{m} e^{2 \delta_{1}+2 \delta_{2}}\left(e^{4 \gamma_{1}}-e^{4 \gamma_{2}}\right)}{2\left(e^{2 \delta_{1}+2 \gamma_{2}}+e^{2 \delta_{2}+2 \gamma_{1}}\right)}, \\
P_{2} & =\frac{\bar{m} e^{2 \delta_{1}+2 \delta_{2}}\left(e^{4 \gamma_{2}}-e^{4 \gamma_{1}}\right)}{2\left(e^{2 \delta_{1}+2 \gamma_{2}}+e^{2 \delta_{2}+2 \gamma_{1}}\right)} .
\end{aligned}
$$

A straightforward calculation shows that if we perform the inversion

$$
r=\frac{\alpha^{2}}{\tilde{r}}, \quad \alpha^{2}=2\left(\bar{m}^{2}+\bar{n}^{2}\right) e^{2\left(\delta_{1}+\delta_{2}+\gamma_{1}+\gamma_{2}\right)},
$$

then $W=W(r)$ satisfies

\footnotetext{
${ }^{3}$ Note that $P_{2}=-P_{1}$ in the static limit. Although this might appear not to be a generic pairwise-equal dyonic configuration, it actually is, once one takes into account that there is an S-duality of the pairwise-equal STU supergravity under which $\left(Q_{1}, P_{1}\right)$ and $\left(Q_{2}, P_{2}\right)$ can both be rotated under
}

$$
\left(\begin{array}{c}
Q_{i} \\
P_{i}
\end{array}\right) \rightarrow\left(\begin{array}{cc}
\cos \theta & \sin \theta \\
-\sin \theta & \cos \theta
\end{array}\right)\left(\begin{array}{c}
Q_{i} \\
P_{i}
\end{array}\right) .
$$

$$
W(r)=\frac{\alpha^{2}}{\tilde{r}^{2}} W(\tilde{r}),
$$

and therefore the metric obeys the conformal inversion symmetry

$$
d s^{2}=\frac{\alpha^{2}}{\tilde{r}^{2}} d \tilde{s}^{2}
$$

where $d \tilde{s}^{2}$ is the same as the original metric $d s^{2}$ given in (3.13), only now written with $\tilde{r}$ in place of $r$.

\section{CONFORMAL INVERSION FOR EIGHT-CHARGE EXTREMAL STATIC STU BLACK HOLES}

The most general black hole solution in STU supergravity carries eight independent charges, namely an electric and a magnetic charge for each of the four electromagnetic fields. The theory has an $S L(2, \mathbb{R})^{3}$ global symmetry, which can be thought of as $S L(2, \mathbb{R})_{1}$ which is an electric/magnetic S-duality; $S L(2, R)_{2}$, which acts on the two-torus when one views the STU supergravity as the $T^{2}$ reduction of the six-dimensional string; and $\operatorname{SL}(2, R)_{3}$, which exchanges Kaluza-Klein and winding fields. The $U(1)^{3}$ compact subgroup of the $S L(2, \mathbb{R})^{3}$ rotates the various charges, while keeping fixed the asymptotic values of the scalar fields. Thus one can always employ the threeparameter $U(1)^{3}$ subgroup to reduce an arbitrary eightcharge black hole to a canonical form in which there are only $5=8-3$ nonvanishing charge parameters.

This reduction to a five-parameter canonical form was employed in [12] in the case of the static BPS extremal STU black holes, in order to construct a solution with five independent charge parameters. They carry charges $(Q, P)$ of the form (after changing to a duality frame that matches the choice in our previous discussions)

$$
\left(Q_{1}, 0\right), \quad\left(Q_{2}, 0\right), \quad\left(Q_{3}, p\right), \quad\left(Q_{4},-p\right) .
$$

For our present purposes it will be more convenient to reintroduce the redundancy of the additional three parameters, so that we can present the static extremal black holes in a symmetrical form with eight independent charge parameters: four electric and four magnetic. In order to do this we shall construct explicitly the action of the $S L(2, \mathbb{R})^{3}$ global symmetry on the STU supergravity fields and the charges, and then make use of the $U(1)^{3}$ compact subgroup in order to derive the general eight-charge solution from the five-parameter solution presented in [12]. Because these steps are a little involved, we relegate them to Appendixes A and B.

The upshot from these calculations is that the metric of the general eight-charge static extremal black hole is 


$$
\begin{aligned}
d s^{2} & =-\frac{r^{2}}{\sqrt{V}} d t^{2}+\frac{\sqrt{V}}{r^{2}}\left(d r^{2}+r^{2} d \Omega^{2}\right), \\
V & =r^{4}+\alpha r^{3}+\beta r^{2}+\gamma r+\Delta
\end{aligned}
$$

where the coefficients $\alpha, \beta, \gamma$, and $\Delta$ are obtained in Eqs. (B8) in Appendix B, and which for convenience we reproduce here:

$$
\begin{aligned}
\alpha= & \sqrt{\left(\sum_{i} Q_{i}\right)^{2}+\left(\sum_{i} P_{i}\right)^{2}}, \\
\beta= & \sum_{i<j}\left(Q_{i} Q_{j}+P_{i} P_{j}\right), \\
\gamma= & \frac{1}{\alpha}\left[4 \Delta+\frac{1}{2} \beta^{2}-\frac{1}{2} \sum_{i<j}\left(P_{i}^{2} P_{j}^{2}+Q_{i}^{2} Q_{j}^{2}+P_{i j} Q_{i j}\right)\right. \\
& \left.-3 \prod_{i} P_{i}-3 \prod_{i} Q_{i}\right], \\
\Delta= & \prod_{i} Q_{i}+\prod_{i} P_{i}+\frac{1}{2} \sum_{i<j} P_{i} Q_{i} P_{j} Q_{j}-\frac{1}{4} \sum_{i} P_{i}^{2} Q_{i}^{2} .
\end{aligned}
$$

In the expression for $\gamma$, we have defined $P_{i j}=P_{i} P_{j}+$ $\left(\prod_{k} P_{k}\right) /\left(P_{i} P_{j}\right)$ and $Q_{i j}=Q_{i} Q_{j}+\left(\prod_{k} Q_{k}\right) /\left(Q_{i} Q_{j}\right)$ (so $P_{12}=P_{1} P_{2}+P_{3} P_{4}$, etc. $)$.

If the radial coordinate of the metric (4.2) is subjected to the inversion

$$
r \rightarrow \tilde{r}=\frac{\sqrt{\Delta}}{r},
$$

then the conformally rescaled metric $d \tilde{s}^{2}$, defined by

$$
d s^{2}=\frac{\sqrt{\Delta}}{\tilde{r}^{2}} d \tilde{s}^{2}, \quad d \tilde{s}^{2}=-\frac{\tilde{r}^{2}}{\sqrt{\tilde{V}}} d t^{2}+\frac{\sqrt{\tilde{V}}}{\tilde{r}^{2}}\left(d \tilde{r}^{2}+\tilde{r}^{2} d \Omega^{2}\right),
$$

where $\tilde{V}=\tilde{r}^{4}+\tilde{\alpha} \tilde{r}^{3}+\tilde{\beta} \tilde{r}^{2}+\tilde{\gamma} \tilde{r}+\tilde{\Delta}$, will be in the same class of black hole metrics provided that there exists a mapping of the charges such that

$$
\tilde{\alpha}=\frac{\gamma}{\sqrt{\Delta}}, \quad \tilde{\beta}=\beta, \quad \tilde{\gamma}=\alpha \sqrt{\Delta}, \quad \tilde{\Delta}=\Delta .
$$

These four equations constitute the conditions that the eight mapped charges $\tilde{Q}_{i}$ and $\tilde{P}_{i}$ must satisfy, if the conformally inverted metric is to be interpretable as again being contained within the eight-charge family of static BPS extremal black holes.

Note that the inversion of the inversion will give back the original metric, up to a constant scale factor that we can always set to unity by normalization. The inversion will then be an involution, and the third equation in (4.6) is automatically satisfied if the first and the fourth are satisfied. Thus we can choose to view the four conditions (4.6) as instead being described by the first, second, and fourth equations in (4.6), together with the normalization condition for the inversion to be an involution.

As one can easily verify, in the special case of the solution with just four electric charges, the expressions (4.3) reduce to

$$
\begin{aligned}
\alpha & =\sum_{i} Q_{i}, & \beta & =\sum_{i<j} Q_{i} Q_{j}, \\
\gamma & =\sum_{i<j<k} Q_{i} Q_{j} Q_{k}, & \Delta & =\prod_{i} Q_{i},
\end{aligned}
$$

and Eqs. (4.6) are indeed all satisfied if the charges are mapped according to the rule (1.12).

For the general eight-charge case, we have not succeeded in finding an elegant formula for a mapping of the charges that satisfies the conditions in (4.6). However, we can see simply by counting the number of conditions, and comparing with the number of unknowns, that we can expect that a solution will always exist. If we include the normalization choice that the inverse of the inverse gives back exactly the original metric then, as indicated in the discussion above, we have a total of four conditions to be satisfied. In the case of the solutions with four electric charges, this meant that the number of conditions was equal to the number of unknowns (the four mapped charge parameters $\tilde{Q}_{i}$, where it was assumed also that the magnetic charges remained zero after the mapping). Thus, in the four-charge case it turned out that there was a unique solution for the mapping of the charges, up to permutations, as given in (1.12).

In the general eight-charge case we still have just four conditions to be satisfied, but we now have eight unknowns (the mapped electric and magnetic charges $\tilde{Q}_{i}$ and $\tilde{P}_{i}$ ). Thus we can expect that not merely will a solution exist for the mapped charges but that there will in fact be a $4=8-4$ parameter family of possible solutions. This perhaps accounts for the difficulty in finding an elegant solution in this case for the mapping of the charges. Unless there exists a criterion for characterizing what constitutes an "elegant" solution, it may not in general be possible to do more than just give the rule "find the four-parameter family of solutions for the mapped charges $\tilde{Q}_{i}$ and $\tilde{P}_{i}$ that solve the four conditions (4.6)."

One way of giving a slightly more constructive description of the mapping of the charges is to consider the set of transformations under the $S L(2, \mathbb{R})^{3}$ global symmetry of the STU theory (see Appendix A). We know that these transformations, implemented as in Eq. (A24), leave the quartic polynomial $\Delta$ defined in the (4.3) invariant, which is precisely what is required by the final equation in (4.6). The other quantities $\alpha, \beta$, and $\gamma$ defined in (4.3) are not invariant under $S L(2, \mathbb{R})^{3}$, although they are invariant 
under the $U(1)^{3}$ compact subgroup. Thus we may view the six-dimensional coset $S L(2, \mathbb{R})^{3} / U(1)^{3}$ as parametrizing candidate transformations of the eight original untilded charges to give the eight tilded charges that obey the remaining three conditions in (4.6).

A relatively simple possibility is to restrict the three $S L(2, \mathbb{R})$ matrices

$$
\left(\begin{array}{ll}
a_{i} & b_{i} \\
c_{i} & d_{i}
\end{array}\right)
$$

to be diagonal,

$$
\left(\begin{array}{cc}
a_{i} & 0 \\
0 & \frac{1}{a_{i}}
\end{array}\right)
$$

for $i=1,2$, and 3 . This then leaves just the three undetermined parameters $a_{i}$ to be solved, by requiring the remaining equations (the first three) in Eq. (4.6) to be satisfied. Provided that there exist real such solutions for the $a_{i}$, then the problem of finding the mapping of charges under the conformal inversion is solved, in the sense that it is reduced to solving three equations for three unknowns.

As a first example, we may consider the four-charge case where the magnetic charges are all zero, which was studied in [4] and is described in the Introduction of the present paper. The mapped charges $\tilde{Q}_{i}$ are given in terms of the original charges $Q_{i}$ by (1.12), and as can easily be checked from the formulas in Appendix A, they are produced by means of the $S L(2, \mathbb{R})^{3}$ transformations with the restricted form (4.9) where

$a_{1}=\sqrt{\frac{Q_{1} Q_{2}}{Q_{3} Q_{4}}}, \quad a_{2}=\sqrt{\frac{Q_{1} Q_{3}}{Q_{2} Q_{4}}}, \quad a_{3}=\sqrt{\frac{Q_{1} Q_{4}}{Q_{2} Q_{3}}}$.

As another example, we may consider the special case of pairwise-equal charges; without loss of generality we choose the case where the gauge fields labeled 1 and 3 are set equal, and likewise the gauge fields labeled 2 and 4. Thus we have

$$
Q_{1}=Q_{3}, \quad Q_{2}=Q_{4}, \quad P_{3}=P_{1}, \quad P_{4}=P_{2} .
$$

The $S L(2, \mathbb{R})^{3}$ global symmetry of the full STU supergravity, described in Appendix A, reduces to just the $S L(2, \mathbb{R})_{2}$ symmetry. In this case the coefficients $\alpha, \beta, \gamma$, and $\Delta$ in (4.3) become

$$
\begin{aligned}
& \alpha=2 \sqrt{\left(P_{1}+P_{2}\right)^{2}+\left(Q_{1}+Q_{2}\right)^{2}}, \quad \beta=\frac{1}{4} \alpha^{2}+2 \sqrt{\Delta}, \\
& \gamma=\alpha \sqrt{\Delta}, \quad \Delta=\left(P_{1} P_{2}+Q_{1} Q_{2}\right)^{2},
\end{aligned}
$$

and so the metric function $V$ becomes the perfect square

$$
V=\left(r^{2}+\frac{\alpha r}{2}+\sqrt{\Delta}\right)^{2} .
$$

After the inversion (4.4) and conformal scaling (4.5), the charge transformation conditions (4.6) reduce simply to

$$
\tilde{\alpha}=\alpha, \quad \tilde{\Delta}=\Delta .
$$

As we mentioned previously, the conformal inversion is actually a symmetry in this pairwise-equal case, and correspondingly, as can be seen from (4.14), one solution for the transformed (tilded) charges is simply to take them to be equal to the original charges. However, it is interesting to note that we can also find other solutions to the conditions (4.14) in which the charges are nontrivially transformed. One way to do this is by taking a diagonal $S L(2, \mathbb{R})_{2}$ transformation with

$$
b_{2}=0, \quad c_{2}=0, \quad d_{2}=\frac{1}{a_{2}} .
$$

From the transformation (A24) we therefore find that Eqs. (4.14) are satisfied if $a_{2}$ is chosen so that

$$
a_{2}^{2}=\frac{Q_{1}^{2}+P_{2}^{2}}{Q_{2}^{2}+P_{1}^{2}} .
$$

The transformed charges are given explicitly by

$\tilde{Q}_{1}=\frac{Q_{1}}{a_{2}}, \quad \tilde{Q}_{2}=a_{2} Q_{2}, \quad \tilde{P}_{1}=a_{2} P_{1}, \quad \tilde{P}_{2}=\frac{P_{2}}{a_{2}}$.

Another, inequivalent, way of solving (4.14) in this pairwise-equal example is to use instead a different noncompact $S L(2, \mathbb{R})_{2}$ transformation, where

$$
\left(\begin{array}{ll}
a_{2} & b_{2} \\
c_{2} & d_{2}
\end{array}\right)=\left(\begin{array}{cc}
\cosh \delta_{2} & \sinh \delta_{2} \\
\sinh \delta_{2} & \cosh \delta_{2}
\end{array}\right) .
$$

From the transformation (A24) we now find that Eqs. (4.14) are satisfied if

$$
\tanh \delta_{2}=\frac{2\left(P_{1} Q_{1}-P_{2} Q_{2}\right)}{P_{1}^{2}+P_{2}^{2}+Q_{1}^{2}+Q_{2}^{2}} .
$$

The transformations of the charges in this case correspond to a different way of solving the constraint equations (4.14). This reflects the fact that the constraints provide an undetermined system of equations. In this pairwise-equal specialization, we have the two constraint equations (4.14) and the four unknowns $\left(\tilde{Q}_{1}, \tilde{Q}_{2}, \tilde{P}_{1}, \tilde{P}_{2}\right)$. The general solution would give a family of transformed charges 
characterized by two continuous parameters. We have exhibited above two discrete members within this family, in addition to the "trivial" member where the charges are untransformed.

In the four-charge and the pairwise-equal examples above, it was possible to present explicit expressions for solutions to the constraint equations. As mentioned previously, this does not appear to be possible in the general case with eight independent charges. For example, we can always look for solutions for the transformed charges by considering the subset of $S L(2, \mathbb{R})^{3}$ transformations described by (4.9). The fourth constraint in (4.6) is automatically satisfied because $\Delta$ is invariant under $S L(2, \mathbb{R})^{3}$, and so the remaining three constraints in (4.6) will imply a discrete set of solutions for the three unknowns $\left(a_{1}, a_{2}, a_{3}\right)$. The three equations are polynomials in the $a_{i}$ parameters, but seemingly they are of too high a degree to be explicitly solvable. Of course, for any specified set of eight original charges one can compute numerically the corresponding $a_{i}$ parameter values that satisfy the constraints, and so in this sense the problem is fully solvable. There is, however, one obstacle that can arise, namely, that it might happen that all of the solutions for the $a_{i}$ parameters turn out to be complex. We have looked at numerous examples of "randomly chosen" sets of original charges $\left(Q_{1}, Q_{2}, Q_{3}, Q_{4}, P_{1}, P_{2}, P_{3}, P_{4}\right)$, and we find that sometimes real solutions for the $a_{i}$ exist, while sometimes only complex solutions exist.

Another option would be to consider the subset of $S L(2, \mathbb{R})^{3}$ transformations where each of the three $S L(2, \mathbb{R})$ elements in the product is of the same form as the $S L(2, \mathbb{R})_{2}$ element (4.18), giving three parameters $\left(\delta_{1}, \delta_{2}, \delta_{3}\right)$ in all. Again, the remaining first three equations in (4.6) would give three equations for the three unknowns $\left(\delta_{1}, \delta_{2}, \delta_{3}\right)$, guaranteeing that solutions would exist. For a given initial choice for the eight charges, it might be that while only complex solutions arose for the three $a_{i}$ parameters in the previous construction, there could be real solutions for the three $\delta_{i}$ parameters in the latter construction. Faute de mieux, one could always, of course, consider the most general possibility of simply viewing the four conditions in (4.6) as providing four equations for eight unknowns. It is still not entirely obvious whether any purely real solutions for the transformed charges must necessarily exist, but with such a large solution space it is perhaps likely. ${ }^{4}$

\footnotetext{
${ }^{4}$ The mapped charges should also, like the original ones, be non-negative, since otherwise the conformally inverted metric $d \tilde{s}^{2}$ defined in (4.5) would have naked singularities outside the horizon at $r=0$. In the examples we found, when the charges come out to be real they are also non-negative. It should be noted also that for the BPS extremal black holes to be regular, without naked singularities, the quartic invariant $\Delta$ should be positive (see, for example, [13]).
}

\section{INVERSION SYMMETRY OF RADIAL EQUATION FOR EIGHT-CHARGE ROTATING BLACK HOLES}

We use the expressions and notation given in the paper [11] by Chow and Compère. It can be seen that the metric will be extremal if $a^{2}=m^{2}+n^{2}$, and then the radial function $R$ will be given by $R=(r-m)^{2}$. We define a new radial coordinate $\rho=r-m$ that vanishes on the horizon. The separation of variables for a solution $\psi$ of $\square \psi=0$ is carried out in [11] by writing $\psi=$ $e^{-\mathrm{i} \omega t+\mathrm{i} k \varphi} \Phi_{r}(r) \Phi_{u}(u)$. Their separated equations for $\Phi_{r}(r)$ and $\Phi_{u}(u)$ are presented in Eq. (9.17) of [11]:

$$
\begin{gathered}
\Phi_{r}^{-1} \frac{d}{d r}\left(R \frac{d \Phi_{r}}{d r}\right)+\frac{\omega^{2} W_{r}^{2}-2 a \omega k L_{r}+a^{2} k^{2}}{R}+C_{c c}=0, \\
\Phi_{u}^{-1} \frac{d}{d u}\left(U \frac{d \Phi_{u}}{d u}\right)-\frac{\omega^{2} W_{u}^{2}+2 a \omega k L_{u}+a^{2} k^{2}}{U}-C_{c c}=0 .
\end{gathered}
$$

[We have renamed their separation constant as $C_{c c}$ rather than $C$, since they already use $C$ for the quantity defined in Eq. (5.5) of [11].] The functions $W_{r}, L_{r}, W_{u}$, and $L_{u}$ are given in [11] (in fact, $L_{u}=0$ when, as in our case, we choose the physical NUT parameter $N$ to be zero). Equations (5.1) are not quite in the form we want, because the constant term in the potential in the equation for $\Phi_{u}(u)$ has dependence on the charge parameters $\delta_{i}$ and $\gamma_{i}$. Since we already know from our results in Sec. II for the generalized Couch-Torrence symmetry of the radial equation for the four-charge solutions that the charge parameters are transformed in the inversion symmetry, we must ensure first in the present eight-charge discussion that the angular equation for $\Phi_{u}$ should be independent of the charge transformations. This is easily achieved, by exploiting the fact that there is always an arbitrariness to shift the separation constant by an additive constant. It is straightforward to see from the definitions in [11] that if we define $\bar{u}=u-n$ and $C_{c c}=-\lambda+4 \omega^{2}\left[n\left(n \mu_{1}-m \mu_{2}\right)+\right.$ $\left.\left(m^{2}+n^{2}\right) C\right]$, then the angular equation becomes

$$
\Phi_{u}^{-1} \frac{d}{d \bar{u}}\left(U \frac{d \Phi_{u}}{d \bar{u}}\right)-\omega^{2} U+\frac{a^{2} k^{2}}{U}+\lambda=0
$$

with $U=a^{2}-\bar{u}^{2}$, and this is completely independent of the charge parameters.

The radial equation now takes the form

$$
\Phi_{r}^{-1} \frac{d}{d \rho}\left(\rho^{2} \frac{d \Phi_{r}}{d \rho}\right)+H(\rho)-\lambda=0
$$

where $H(\rho)$ is given by 


$$
\begin{aligned}
H(\rho)= & \omega^{2}\left\{\rho^{2}+\left(2 a^{2}\left(\nu_{2}+2 D\right)-\frac{a k}{\omega}\right)^{2} \rho^{-2}\right\} \\
& +4 \omega^{2}\left\{M \rho+\frac{a^{2} \nu_{2}}{m}\left(2 a^{2}\left(\nu_{2}+2 D\right)-\frac{a k}{\omega}\right) \rho^{-1}\right\} \\
& +4 \omega^{2} a^{2}\left(\mu_{1}+C+\nu_{1}^{2}+\nu_{2}^{2}\right),
\end{aligned}
$$

and the various quantities $\nu_{1}, \nu_{2}, \mu_{1}, C, D$, and $M$ are defined in [11].

We may now seek an inversion symmetry of the radial equation. Thus we transform to a new radial coordinate $\tilde{\rho}$ such that

$$
\rho=\frac{\beta^{2}}{\tilde{\rho}},
$$

where $\beta$ is a constant to be determined, and test to see whether

$$
H(\rho)=\tilde{H}(\tilde{\rho}),
$$

where the function $\tilde{H}$ is the same in form as the function $H$ given in (5.4), but using redefined charge parameters. ${ }^{5}$ Thus we have

$$
\begin{aligned}
H(\rho)= & \omega^{2}\left\{\beta^{4} \tilde{\rho}^{-2}+\left(2 a^{2}\left(\nu_{2}+2 D\right)-\frac{a k}{\omega}\right)^{2} \beta^{-4} \tilde{\rho}^{2}\right\} \\
& +4 \omega^{2}\left\{M \beta^{2} \tilde{\rho}^{-1}+\frac{a^{2} \nu_{2}}{m}\left(2 a^{2}\left(\nu_{2}+2 D\right)-\frac{a k}{\omega}\right) \beta^{-2} \tilde{\rho}\right\} \\
& +4 \omega^{2} a^{2}\left(\mu_{1}+C+\nu_{1}^{2}+\nu_{2}^{2}\right) .
\end{aligned}
$$

Assuming that $\beta$ is universal, that is to say, that it is invariant under the transformation of the charge parameters, we then have, by comparing the various powers of $\rho$ or $\tilde{\rho}$ in the proposed relation (5.6) that

$$
\begin{aligned}
& \rho^{-2}: \beta^{4}=\left(2 a^{2}\left(\nu_{2}+2 D\right)-\frac{a k}{\omega}\right)^{2}, \\
& \rho^{2}: \beta^{4}=\left(2 \tilde{a}^{2}\left(\tilde{\nu}_{2}+2 \tilde{D}\right)-\frac{\tilde{a} k}{\omega}\right)^{2}, \\
& \rho^{-1}: \tilde{M} \beta^{2}=\frac{a^{2} \nu_{2}}{m}\left(2 a^{2}\left(\nu_{2}+2 D\right)-\frac{a k}{\omega}\right), \\
& \rho: M \beta^{2}=\frac{\tilde{a}^{2} \tilde{\nu}_{2}}{\tilde{m}}\left(2 \tilde{a}^{2}\left(\tilde{\nu}_{2}+2 \tilde{D}\right)-\frac{\tilde{a} k}{\omega}\right), \\
& \rho^{0}: \mu_{1}+C+\nu_{1}^{2}+\nu_{2}^{2}=\tilde{\mu}_{1}+\tilde{C}+\tilde{\nu}_{1}^{2}+\tilde{\nu}_{2}^{2} .
\end{aligned}
$$

From the first two equations in (5.8) we have

\footnotetext{
${ }^{5}$ This, at least, is what we found for the four-charge rotating solutions in Sec. II; the potential in the radial equation was related under inversion to a potential of the same form but with redefined charge parameters.
}

$\beta^{2}=2 a^{2}\left(\nu_{2}+2 D\right)-\frac{a k}{\omega}=2 \tilde{a}^{2}\left(\tilde{\nu}_{2}+2 \tilde{D}\right)-\frac{\tilde{a} k}{\omega}$.

Since we are assuming $\omega$ does not transform, and since this relation should hold for all frequencies $\omega$, it follows that

$$
\tilde{a}=a, \quad \tilde{\nu}_{2}+2 \tilde{D}=\nu_{2}+2 D .
$$

(Note that we are not making any assumption about $m$ or $n$ being invariant under the transformation.) The third and fourth equations in (5.8) then imply

$$
\tilde{M}=\frac{a^{2} \nu_{2}}{m}, \quad M=\frac{a^{2} \tilde{\nu}_{2}}{\tilde{m}} .
$$

From [11], $M=m \mu_{1}+n \mu_{2}$, and since we must set $n=$ $-m \nu_{1} / \nu_{2}$ so that the physical NUT charge $N=m \nu_{1}+n \nu_{2}$ is zero, we have

$$
M=\frac{m}{\nu_{2}}\left(\mu_{1} \nu_{2}-\mu_{2} \nu_{1}\right),
$$

together with the transformed version where all quantities are tilded. Equations (5.11) therefore imply

$a^{2} \nu_{2} \tilde{\nu}_{2}=m \tilde{m}\left(\mu_{1} \nu_{2}-\mu_{2} \nu_{2}\right)=m \tilde{m}\left(\tilde{\mu}_{1} \tilde{\nu}_{2}-\tilde{\mu}_{2} \tilde{\nu}_{2}\right)$,

and hence

$$
\mu_{1} \nu_{2}-\mu_{2} \nu_{2}=\tilde{\mu}_{1} \tilde{\nu}_{2}-\tilde{\mu}_{2} \tilde{\nu}_{2} .
$$

Collecting the results so far, we see from the last equation in (5.8), from (5.10), and from (5.14) that the three quantities

$$
\begin{aligned}
& X_{1} \equiv \mu_{1}+C+\nu_{1}^{2}+\nu_{2}^{2}, \quad X_{2} \equiv \nu_{2}+2 D, \\
& X_{3} \equiv \mu_{1} \nu_{2}-\mu_{2} \nu_{2}
\end{aligned}
$$

should all be invariant under the transformation of the charge parameters that accompanies the inversion (5.5). A natural guess for the inversion transformation, which would reduce to the known four-charge case $\gamma_{i}=0$ discussed in Sec. II (and its duality partner where instead $\delta_{i}=0$ ) and would also reduce to the known pairwise-equal dyonic case discussed in Sec. III, is to try

$$
\tilde{\delta}_{i}=-\delta_{i}+\frac{1}{2} \sum_{j} \delta_{j}, \quad \tilde{\gamma}_{i}=-\gamma_{i}+\frac{1}{2} \sum_{j} \gamma_{j} .
$$

One can, in fact, verify that the three quantities $X_{1}, X_{2}$, and $X_{3}$ defined in (5.15) are indeed invariant under (5.16). However, there is one further condition contained in the set of Eqs. (5.8), since until now we just extracted the one condition (5.14) from the third and fourth equations in (5.8). The remaining condition can be found by noting that 
the extremality condition $a^{2}=m^{2}+n^{2}$ implies $a^{2}=$ $m^{2}\left(1+\nu_{1}^{2} / \nu_{2}^{2}\right)$ (and its tilded version), and using this in the third and fourth equations of (5.8) leads to

$$
\left(\nu_{1}^{2}+\nu_{2}^{2}\right)\left(\tilde{\nu}_{1}^{2}+\tilde{\nu}_{2}^{2}\right)=X_{3}^{2}
$$

Straightforward calculation reveals that while this is indeed consistent with (5.16) in the four-charge specialization or in the pairwise-equal specialization (as it must be, since those cases were already fully verified), it is not consistent in the general eight-charge case. Thus the transformation of the $\delta_{i}$ and $\gamma_{i}$ charge parameters in the general case must be more complicated than the guess in (5.16). However, since the number of conditions that must be satisfied in order to achieve $H(\rho)=\tilde{H}(\tilde{\rho})$ is smaller than the number of unknown transformed charge parameters $\left(\tilde{\delta}_{i}, \tilde{\gamma}_{i}\right)$, we can conclude that it must be possible to solve for such $\left(\tilde{\delta}_{i}, \tilde{\gamma}_{i}\right)$, even if we cannot present the solution in a universal and elegant form.

\section{CONCLUDING REMARKS}

Studies of general rotating black holes in maximally supersymmetric ungauged supergravity theories (often referred to as STU black holes) revealed their numerous intriguing properties which often stem from, and provide an intriguing generalization of, properties of Kerr-Newman black holes in Einstein-Maxwell gravity. Furthermore, the extremal black holes of that type are endowed with further enhanced symmetry properties, again generalizing those of extremal Kerr-Newman black holes. For example, there has been substantial progress in recent studies of the Aretakis charge for both extremal Reissner-Nordström black holes [5,6] and extremal Kerr black holes [14] as well as recent generalizations to extremal static four-charge black holes [4] and rotating ones [15] in STU supergravity. Furthermore, in [15] Aretakis charges for five-dimensional extremal STU black holes were derived, while generalizations to related conserved charges of extremal static $p$-branes were given in [16].

In this paper we focused principally on another type of symmetry, namely the Couch-Torrence symmetry of the radial part of the massless Klein-Gordon equation for extremal rotating STU black holes. This symmetry [1] was originally obtained for the radial equation of the extremal Reissner-Nordström metric under the conformal inversion transformation. The generalization of the symmetry to extremal rotating black holes, such as KerrNewman ones, is possible due to the separability of the massless Klein-Gordon equation in these backgrounds, where the inversion transformation of the radial equation depends not only on the black hole charge and the rotation parameters, but also on the mode eigenvalues for energy $\omega$ and azimuthal angular momentum $m$.
In the present paper we further generalized this symmetry from the extremal static four-charge black holes [4] to the extremal rotating four-charge black holes in STU supergravity. We showed that in this case the radial equation for the separable massless Klein-Gordon equation indeed exhibits an inversion symmetry, where again the inversion radius depends not only on the black hole charges and rotation parameter, but also on the mode eigenvalues $\omega$ and $m$. However, unlike in the Kerr-Newman case, after the transformation the radial equation is different from the original one, in the sense that it is the radial equation for a transformed set of the four charge parameters. This is a natural generalization of the properties of the static fourcharge STU extremal black holes, where a given black hole is mapped under conformal inversion to another member of the four-charge family with a transformed set of electric charges [4].

We also investigated the conformal inversion symmetry for the most general extremal BPS black holes in STU supergravity, specified by eight charges, in both the static and the rotating cases. For the static eight-charge case, which corresponds to BPS solutions, we showed that the entire family of black holes maps into itself under conformal inversion. However, unlike in the four-charge solutions, we were unable to give an elegant formula for the mapping of the eight charges under conformal inversion. This is related to the fact that the number of equations comprising the conditions for conformal inversion invariance is less than the number of unknowns (the eight mapped charges), and thus there is no unique solution.

In the case of extremal eight-charge rotating solutions, just as in the four-charge case, the massless Klein-Gordon equation is separable and the behavior of the radial wave equation under inversion can be investigating along the same lines. However, just as in the static case, the inversion symmetry conditions are underdetermined; i.e., they do not fully constrain the mapped charges. ${ }^{6}$ We have provided a systematic procedure, which employs the action of the coset generators of $\prod_{i=1}^{3} S L(2, \mathbb{R})_{i} / U(1)_{i}$ on the eight charges, in order to solve the constraints (4.6) for the mapped charges. Further investigations of these transformations are in order.

The generalization of the Couch-Torrence symmetry to general extremal rotating black holes of STU supergravity demonstrates the existence of another symmetry of general asymptotically flat black holes, extending the symmetries of black holes in Einstein-Maxwell gravity. In spite of the significantly more complicated field content and the structure of the Lagrangian, which results in significantly more complicated metrics for the black holes, it is

\footnotetext{
${ }^{6}$ In the extremal eight-charge rotating case there is also another, non-BPS, branch. The extremal Kaluza-Klein dyon is a particular example of that type, which by itself does not possess the inversion symmetry, but should be mapped on to an orbit of the eight-charge non-BPS branch.
} 
interesting that the conformal inversion symmetry of the radial part of the massless Klein-Gordon equation persists in its generalized form.

We expect that a generalization of the Couch-Torrence symmetry may persist also for general extremal black holes of STU supergravity in five dimensions. Again the separability of the massless Klein-Gordon equation will play an important role. We defer further consideration of this case to future work.

We would like to conclude by emphasizing that our analysis focused on conformal inversion transformations for extremal BPS black holes in STU supergravity for which the asymptotic values of the scalar fields were set to zero. It would, of course, be interesting to study conformal inversion transformations for such black holes with nonzero asymptotic values of the scalar fields. This explicit dependence on the asymptotic scalar fields is currently being investigated [17]. Such generalizations of the black hole solutions would in turn allow for generalizations of the Couch-Torrence transformations that could also involve scalar field transformations. Furthermore, one would be able to address other types of inversion transformations, including those studied in [18].

\section{ACKNOWLEDGMENTS}

We are grateful to Hadi Godazgar, Mahdi Godazgar, and Alejandro Satz for helpful discussions. The work of M. C. is supported in part by the DOE (HEP) Award No. DESC0013528, the Fay R. and Eugene L. Langberg Endowed Chair (M. C.), and the Slovenian Research Agency (ARRS No. P1-0306). The work of C. N.P. is supported in part by DOE Grant No. DE-FG02-13ER42020.

\section{APPENDIX A: BOSONIC LAGRANGIAN OF STU SUPERGRAVITY IN SYMMETRICAL FORM}

Here, we present the bosonic sector of the STU supergravity Lagrangian in a form where the four field strengths enter symmetrically. We take the Lagrangian as given in Appendix B of [19], except that the field strengths called $F^{+}$in that paper will be called $F^{-}$here, to match conveniently with the conventions of Freedman and Van Proeyen [20].

In the notation of [20], but written in the language of differential forms, their Eq. (4.66) becomes

$$
\mathcal{L}(F)=-\frac{1}{2} f_{A B}^{R} * F^{A} \wedge F^{B}+\frac{\mathrm{i}}{2} f_{A B}^{I} * \tilde{F}^{A} \wedge F^{B},
$$

where $f_{A B}^{R}$ and $f_{A B}^{I}$ denote the real and imaginary parts of $f_{A B}$ (i.e., $f_{A B}=f_{A B}^{R}+\mathrm{i} f_{A B}^{I}$ ). Now $\tilde{F} \equiv-\mathrm{i} * F$, and $*^{2}=$ -1 in four-dimensional spacetime when acting on 2-forms, so $* \tilde{F}=$ i $F$; hence we have

$$
\mathcal{L}(F)=-\frac{1}{2} f_{A B}^{R} * F^{A} \wedge F^{B}-\frac{1}{2} f_{A B}^{I} F^{A} \wedge F^{B}
$$

Note that the field strengths $F^{A}$ are simply the exterior derivatives of potentials, $F^{A}=d A^{A}$. Note also that $f_{A B}$ is symmetric in $A$ and $B$.

As in [20] we define $F^{ \pm}=\frac{1}{2}(F \pm \tilde{F})$, and hence

$$
F^{ \pm}=\frac{1}{2}(F \mp \mathrm{i} * F), \quad * F^{ \pm}= \pm \mathrm{i} F^{ \pm} .
$$

Noting that for any 2-forms $X$ and $Y$ we have

$$
\frac{1}{2} X^{\mu \nu} Y_{\mu \nu} * \mathbb{1}=* X \wedge Y=* Y \wedge X,
$$

we see that the wedge product of any self-dual 2 -form with any anti-self-dual 2-form is zero:

$$
\begin{aligned}
F^{+} \wedge F^{-} & =-\mathrm{i} * F^{+} \wedge F^{-}=-\mathrm{i} * F^{-} \wedge F^{+}=-F^{-} \wedge F^{+} \\
& =-F^{+} \wedge F^{-}
\end{aligned}
$$

hence $F^{+} \wedge F^{-}=0$. From (A3) we have

$$
F=F^{+}+F^{-}, \quad * F=\mathrm{i}\left(F^{+}-F^{-}\right) .
$$

From the above, it follows that the Lagrangian (A2) can be written as

$$
\mathcal{L}(F)=\frac{\mathrm{i}}{2} f_{A B} F^{-A} \wedge F^{-B}-\frac{\mathrm{i}}{2} \bar{f}_{A B} F^{+A} \wedge F^{+B},
$$

and hence

$$
\mathcal{L}(F)=-\frac{1}{2} f_{A B} * F^{-A} \wedge F^{-B}-\frac{1}{2} \bar{f}_{A B} * F^{+A} \wedge F^{+B} .
$$

In this form, we can compare with Eq. (B.7) in [19] (with the understanding that the roles of + and - superscripts on $F$ are exchanged as mentioned previously), and hence read off from (B.9) of [19] that the matrix $f$, with components $f_{A B}$, is given by

$f=\frac{1}{W}\left(\begin{array}{cccc}e^{-\lambda_{1}} & e^{\varphi_{1}} \beta_{1} & e^{\varphi_{2}} \beta_{2} & e^{\varphi_{3}} \beta_{3} \\ e^{\varphi_{1}} \beta_{1} & e^{-\lambda_{2}} \alpha_{2} \alpha_{3} & -e^{-\varphi_{3}} \alpha_{3} \beta_{3} & -e^{-\varphi_{2}} \alpha_{2} \beta_{2} \\ e^{\varphi_{2}} \beta_{2} & -e^{-\varphi_{3}} \alpha_{3} \beta_{3} & e^{-\lambda_{3}} \alpha_{1} \alpha_{3} & -e^{-\varphi_{1}} \alpha_{1} \beta_{1} \\ e^{\varphi_{3}} \beta_{3} & -e^{-\varphi_{2}} \alpha_{2} \beta_{2} & -e^{-\varphi_{1}} \alpha_{1} \beta_{1} & e^{-\lambda_{4}} \alpha_{1} \alpha_{2}\end{array}\right)$,

where 


$$
\begin{aligned}
& \lambda_{1}=-\varphi_{1}-\varphi_{2}-\varphi_{3}, \quad \lambda_{2}=-\varphi_{1}+\varphi_{2}+\varphi_{3}, \quad \lambda_{3}=\varphi_{1}-\varphi_{2}+\varphi_{3}, \quad \lambda_{4}=\varphi_{1}+\varphi_{2}-\varphi_{3}, \\
& \alpha_{i}=1+e^{2 \varphi_{i}} \chi_{i}^{2}, \\
& \beta_{1}=e^{\varphi_{2}+\varphi_{3}} \chi_{2} \chi_{3}+\mathrm{i} e^{\varphi_{1}} \chi_{1}, \quad \beta_{2}=e^{\varphi_{1}+\varphi_{3}} \chi_{1} \chi_{3}+\mathrm{i} e^{\varphi_{2}} \chi_{2}, \quad \beta_{3}=e^{\varphi_{1}+\varphi_{2}} \chi_{1} \chi_{2}+\mathrm{i} e^{\varphi_{3}} \chi_{3}, \\
& W=1+\sum_{i} e^{2 \varphi_{i}} \chi_{i}^{2}-2 \mathrm{i} e^{\varphi_{1}+\varphi_{2}+\varphi_{3}} \chi_{1} \chi_{2} \chi_{3} .
\end{aligned}
$$

The field equations following from (A2) are $d G_{A}=0$, where the 2-forms $G^{A}$ are read off from varying $\mathcal{L}(F)$ with respect to $F^{A}$ :

$$
\delta \mathcal{L}(F)=G_{A} \delta F^{A}=-f_{A B}^{R} * F^{B}-f_{A B}^{I} F^{B},
$$

and so

$$
G_{A}=-f_{A B}^{R} * F^{B}-f_{A B}^{I} F^{B} .
$$

It then follows that $G_{A}^{ \pm} \equiv \frac{1}{2}\left(G_{A} \mp \mathrm{i} * G_{A}\right)$ are given by

$$
G_{A}^{-}=\mathrm{i} f_{A B} F^{-B}, \quad G_{A}^{+}=-\mathrm{i} f_{A B}^{*} F^{+B} .
$$

Note that the Bianchi identities $d F^{A}=0$ and the field equations $d G_{A}=0$ can be written as

$$
d \Im\left(* F^{ \pm}\right)=0, \quad d \Im\left(* G^{ \pm}\right)=0
$$

( $\Re$ and $\Im$ denote the real and imaginary parts.)

The Bianchi equations and equations of motion are invariant under the transformations

$$
\begin{gathered}
\qquad\left(\begin{array}{l}
F^{ \pm} \\
G^{ \pm}
\end{array}\right) \rightarrow\left(\begin{array}{l}
F^{ \pm \prime} \\
G^{ \pm \prime}
\end{array}\right)=\mathcal{S}\left(\begin{array}{l}
F^{ \pm} \\
G^{ \pm}
\end{array}\right), \\
\text {hence }\left(\begin{array}{l}
F \\
G
\end{array}\right) \rightarrow\left(\begin{array}{l}
F^{\prime} \\
G^{\prime}
\end{array}\right)=\mathcal{S}\left(\begin{array}{l}
F \\
G
\end{array}\right),
\end{gathered}
$$

where

$$
\mathcal{S}=\left(\begin{array}{ll}
A & B \\
C & D
\end{array}\right)
$$

where $A, B, C$, and $D$ are real constant $4 \times 4$ matrices, provided that the scalar fields transform appropriately: We have

$$
G^{-\prime}=(C+\mathrm{i} D f) F^{-}=(C+\mathrm{i} D f)(A+\mathrm{i} B f)^{-1} F^{-\prime} .
$$

Since the transformed fields must also obey (A13), this implies that the scalar matrix $f$ must transform according to [20]

$$
\mathrm{i} f^{\prime}=(C+\mathrm{i} D f)(A+\mathrm{i} B f)^{-1} .
$$

It is important that $f^{\prime}$, like $f$, must be symmetric, and so this implies that the matrices $A, B, C$, and $D$ must obey the relations

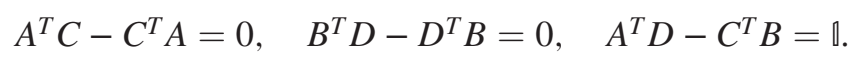

These are precisely the conditions for the matrix $\mathcal{S}$ defined in (A16) to be an element of $S p(8, \mathbb{R})$, obeying [20]

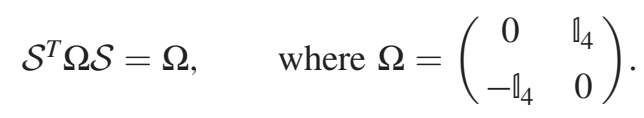

The scalar field Lagrangian is

$$
\mathcal{L}(\varphi, \chi)=-\frac{1}{2} \sum_{i}\left(* d \varphi_{i} \wedge d \varphi_{i}+e^{2 \varphi_{i}} * d \chi_{i} \wedge d \chi_{i}\right),
$$

and this is invariant under the $S L(2, \mathbb{R})_{1} \times S L(2, \mathbb{R})_{2} \times$ $S L(2, \mathbb{R})_{3}$, where the three $S L(2, \mathbb{R})$ act in the standard way:

$$
\tau_{i} \rightarrow \tau_{i}^{\prime}=\frac{a_{i} \tau_{i}+b_{i}}{c_{i} \tau_{i}+d_{i}}
$$

with $a_{i} d_{i}-b_{i} c_{i}=1$ for each $i$, and

$$
\tau_{i}=\chi_{i}+\mathrm{i} e^{-\varphi_{i}}
$$

Thus the entire STU supergravity theory is invariant under the intersection of the $\operatorname{Sp}(8, \mathbb{R})$ symmetry of the fieldstrength sector and the $S L(2, \mathbb{R})_{1} \times S L(2, \mathbb{R})_{2} \times S L(2, \mathbb{R})_{3}$ symmetry of the scalar sector. That is to say, the theory (at the level of the equations of motion) is invariant under $S L(2, \mathbb{R})_{1} \times S L(2, \mathbb{R})_{2} \times S L(2, \mathbb{R})_{3}$.

To see how the field strengths and their duals transform under the $S L(2, \mathbb{R})_{1} \times S L(2, \mathbb{R})_{2} \times S L(2, \mathbb{R})_{3}$ symmetry, we just have to work out the $A, B, C$, and $D$ matrices for which the transformation (A18) of the scalar field matrix matches with the $S L(2, \mathbb{R})_{1} \times S L(2, \mathbb{R})_{2} \times S L(2, \mathbb{R})_{3}$ transformations (A22). After some algebra, we find 


$$
\begin{aligned}
& A=\left(\begin{array}{cccc}
a_{1} a_{2} a_{3} & -a_{1} b_{2} b_{3} & -b_{1} a_{2} b_{3} & -b_{1} b_{2} a_{3} \\
-a_{1} c_{2} c_{3} & a_{1} d_{2} d_{3} & b_{1} c_{2} d_{3} & b_{1} d_{2} c_{3} \\
-c_{1} a_{2} c_{3} & c_{1} b_{2} d_{3} & d_{1} a_{2} d_{3} & d_{1} b_{2} c_{3} \\
-c_{1} c_{2} a_{3} & c_{1} d_{2} b_{3} & d_{1} c_{2} b_{3} & d_{1} d_{2} a_{3}
\end{array}\right), \quad B=\left(\begin{array}{ccccc}
b_{1} b_{2} b_{3} & -b_{1} a_{2} a_{3} & -a_{1} b_{2} a_{3} & -a_{1} a_{2} b_{3} \\
-b_{1} d_{2} d_{3} & b_{1} c_{2} c_{3} & a_{1} d_{2} c_{3} & a_{1} c_{2} d_{3} \\
-d_{1} b_{2} d_{3} & d_{1} a_{2} c_{3} & c_{1} b_{2} c_{3} & c_{1} a_{2} d_{3} \\
-d_{1} d_{2} b_{3} & d_{1} c_{2} a_{3} & c_{1} d_{2} a_{3} & c_{1} c_{2} b_{3}
\end{array}\right), \\
& C=\left(\begin{array}{cccc}
c_{1} c_{2} c_{3} & -c_{1} d_{2} d_{3} & -d_{1} c_{2} d_{3} & -d_{1} d_{2} c_{3} \\
-c_{1} a_{2} a_{3} & c_{1} b_{2} b_{3} & d_{1} a_{2} b_{3} & d_{1} b_{2} a_{3} \\
-a_{1} c_{2} a_{3} & a_{1} d_{2} b_{3} & b_{1} c_{2} b_{3} & b_{1} d_{2} a_{3} \\
-a_{1} a_{2} c_{3} & a_{1} b_{2} d_{3} & b_{1} a_{2} d_{3} & b_{1} b_{2} c_{3}
\end{array}\right), \quad D=\left(\begin{array}{cccc}
d_{1} d_{2} d_{3} & -d_{1} c_{2} c_{3} & -c_{1} d_{2} c_{3} & -c_{1} c_{2} d_{3} \\
-d_{1} b_{2} b_{3} & d_{1} a_{2} a_{3} & c_{1} b_{2} a_{3} & c_{1} a_{2} b_{3} \\
-b_{1} d_{2} b_{3} & b_{1} c_{2} a_{3} & a_{1} d_{2} a_{3} & a_{1} c_{2} b_{3} \\
-b_{1} b_{2} d_{3} & b_{1} a_{2} c_{3} & a_{1} b_{2} c_{3} & a_{1} a_{2} d_{3}
\end{array}\right) .
\end{aligned}
$$

Note that $S L(2, \mathbb{R})^{3}$ transformation matrix $S$ factorizes into the (commuting) product of factors, $S=S_{1} S_{2} S_{3}$, where $S_{i}$ is the transformation matrix for the $i$ th $S L(2, \mathbb{R})$, which can be written in the form (A16) for very simple $4 \times 4$ blocks $A_{i}, B_{i}, C_{i}$, and $D_{i}$. These can be read off from the general $A$, $B, C$, and $D$ matrices above by setting $a_{j}=1, b_{j}=0$, $c_{j}=0$, and $d_{j}=1$ for the two values of $j$ that are not equal to $i$. For example,

$$
\begin{aligned}
A_{1} & =\left(\begin{array}{cccc}
a_{1} & 0 & 0 & 0 \\
0 & a_{1} & 0 & 0 \\
0 & 0 & d_{1} & 0 \\
0 & 0 & 0 & d_{1}
\end{array}\right), \quad B_{1}=\left(\begin{array}{cccc}
0 & -b_{1} & 0 & 0 \\
-b_{1} & 0 & 0 & 0 \\
0 & 0 & 0 & c_{1} \\
0 & 0 & c_{1} & 0
\end{array}\right), \\
C_{1} & =\left(\begin{array}{cccc}
0 & -c_{1} & 0 & 0 \\
-c_{1} & 0 & 0 & 0 \\
0 & 0 & 0 & b_{1} \\
0 & 0 & b_{1} & 0
\end{array}\right),
\end{aligned}
$$

The magnetic and electric charges transform according to

$$
\left(\begin{array}{l}
\mathbf{P}^{\prime} \\
\mathbf{Q}^{\prime}
\end{array}\right)=\mathcal{S}\left(\begin{array}{l}
\mathbf{P} \\
\mathbf{Q}
\end{array}\right)
$$

where

$$
\mathbf{P}=\left(\begin{array}{c}
P_{1} \\
P_{2} \\
P_{3} \\
P_{4}
\end{array}\right), \quad \mathbf{Q}=\left(\begin{array}{c}
Q_{1} \\
Q_{2} \\
Q_{3} \\
Q_{4}
\end{array}\right)
$$

The $U(1)^{3}$ compact subgroup of the full $S L(2, \mathbb{R})^{3}$ symmetry group corresponds to taking

$$
\left(\begin{array}{ll}
a_{i} & b_{i} \\
c_{i} & d_{i}
\end{array}\right)=\left(\begin{array}{cc}
\cos \theta_{i} & \sin \theta_{i} \\
-\sin \theta_{i} & \cos \theta_{i}
\end{array}\right)
$$

\section{APPENDIX B: EIGHT-CHARGE STATIC BLACK HOLE}

The five-charge static black hole constructed in [12] has metric given by

$$
d s^{2}=-\frac{r^{2}}{\sqrt{V}} d t^{2}+\frac{\sqrt{V}}{r^{2}}\left(d r^{2}+r^{2} d \Omega^{2}\right)
$$

where

$$
\begin{aligned}
V= & \left(r+Q_{1}\right)\left(r+Q_{2}\right)\left(r+Q_{3}\right)\left(r+Q_{4}\right) \\
& -p^{2}\left[r+\frac{1}{2}\left(Q_{3}+Q_{4}\right)\right]^{2} \\
= & r^{4}+\alpha r^{3}+\beta r^{2}+\gamma r+\Delta,
\end{aligned}
$$

with

$$
\begin{aligned}
\alpha & =\sum_{i} Q_{i}, \quad \beta=\sum_{i<j} Q_{i} Q_{j}-p^{2}, \\
\gamma & =\sum_{i<j<k} Q_{i} Q_{j} Q_{k}-p^{2}\left(Q_{3}+Q_{4}\right), \\
\Delta & =\prod_{i} Q_{i}-\frac{1}{4} p^{2}\left(Q_{3}+Q_{4}\right)^{2} .
\end{aligned}
$$

These five-charge black holes correspond to restricting the charges $P_{i}$ and $Q_{i}$ of a general eight-charge static BPS extremal STU black hole by specializing the magnetic charges to

$P_{1}=0, \quad P_{2}=0, \quad P_{3}=p, \quad P_{4}=-p$

as in (4.1). To obtain the expressions for the coefficients $\alpha$, $\beta, \gamma$, and $\Delta$ for the general case with eight independent charges, we may act on the restricted five-charge solution with the $U(1)^{3}$ compact subgroup of the $S L(2, R)^{3}$ global symmetry. The eight parameters of the general solution then correspond to the original five charge parameters plus the three parameters of the $U(1)^{3}$ rotations. Conversely, we can determine the three $U(1)^{3}$ rotation angles $\theta_{1}, \theta_{2}$, and $\theta_{3}$ such that the acting on a general eight-charge configuration as in (A24) gives primed charges that are subject to the fivecharge specialization in (B4), where we restrict to the $U(1)^{3}$ subgroup as defined in (A26). Defining 


$$
\theta_{ \pm}=\theta_{2} \pm \theta_{3}
$$

we find

$$
\begin{aligned}
\tan \theta_{+} & =\frac{\left(P_{1}+P_{2}\right)-\left(Q_{1}+Q_{2}\right) \tan \theta_{1}}{\left(Q_{3}+Q_{4}\right)+\left(P_{3}+P_{4}\right) \tan \theta_{1}}, \quad \tan \theta_{-}=\frac{\left(P_{1}-P_{2}\right)+\left(Q_{1}-Q_{2}\right) \tan \theta_{1}}{\left(Q_{3}-Q_{4}\right)-\left(P_{3}-P_{4}\right) \tan \theta_{1}}, \\
\tan 2 \theta_{1} & =\frac{2\left(P_{3}+P_{4}\right)\left(Q_{1}+Q_{2}\right)-2\left(P_{1}+P_{2}\right)\left(Q_{3}+Q_{4}\right)}{\left(P_{1}+P_{2}+P_{3}+P_{4}\right)\left(P_{1}+P_{2}-P_{3}-P_{4}\right)+\left(Q_{1}+Q_{2}+Q_{3}+Q_{4}\right)\left(Q_{1}+Q_{2}-Q_{3}-Q_{4}\right)} .
\end{aligned}
$$

It is also helpful to note that

$$
\tan \left(\theta_{1}+\theta_{2}+\theta_{3}\right)=\frac{P_{1}+P_{2}+P_{3}+P_{4}}{Q_{1}+Q_{2}+Q_{3}+Q_{4}} .
$$

After some algebra, we can now read off the general expressions for the coefficients in (B3) for the general eight-charge solutions. We find

$$
\begin{aligned}
\alpha & =\sqrt{\left(\sum_{i} Q_{i}\right)^{2}+\left(\sum_{i} P_{i}\right)^{2}}, \\
\beta & =\sum_{i<j}\left(Q_{i} Q_{j}+P_{i} P_{j}\right), \\
\gamma & =\frac{1}{\alpha}\left[4 \Delta+\frac{1}{2} \beta^{2}-\frac{1}{2} \sum_{i<j}\left(P_{i}^{2} P_{j}^{2}+Q_{i}^{2} Q_{j}^{2}+P_{i j} Q_{i j}\right)-3 \prod_{i} P_{i}-3 \prod_{i} Q_{i}\right], \\
\Delta & =\prod_{i} Q_{i}+\prod_{i} P_{i}+\frac{1}{2} \sum_{i<j} P_{i} Q_{i} P_{j} Q_{j}-\frac{1}{4} \sum_{i} P_{i}^{2} Q_{i}^{2} .
\end{aligned}
$$

In the expression for $\gamma$, we have defined $P_{i j}=P_{i} P_{j}+$ $\left(\prod_{k} P_{k}\right) /\left(P_{i} P_{j}\right)$ and $Q_{i j}=Q_{i} Q_{j}+\left(\prod_{k} Q_{k}\right) /\left(Q_{i} Q_{j}\right)$ (so $P_{12}=P_{1} P_{2}+P_{3} P_{4}$, etc.).

Note that $\Delta$ in (B8) is the standard quartic invariant, and it is invariant under the full $\operatorname{SL}(2, R)^{3}$ global symmetry group of the STU theory. The coefficients $\alpha, \beta$, and $\gamma$ in (B8) are only invariant under the $U(1)^{3}$ subgroup. This can be understood from the fact that the solutions have been chosen so that the scalar fields all go to zero at infinity. Only the $U(1)^{3}$ subgroup of $S L(2, R)^{3}$ has the property of preserving this asymptotic condition on the scalars.

If the radial coordinate of the static metric is subjected to the inversion then the conformally rescaled metric $d \tilde{s}^{2}$, defined by

$d s^{2}=\frac{\sqrt{\Delta}}{\tilde{r}^{2}} d \tilde{s}^{2}, \quad d \tilde{s}^{2}=-\frac{\tilde{r}^{2}}{\sqrt{\tilde{V}}} d t^{2}+\frac{\sqrt{\tilde{V}}}{\tilde{r}^{2}}\left(d \tilde{r}^{2}+\tilde{r}^{2} d \Omega^{2}\right)$,

where $\tilde{V}=\tilde{r}^{4}+\tilde{\alpha} \tilde{r}^{3}+\tilde{\beta} \tilde{r}^{2}+\tilde{\gamma} \tilde{r}+\tilde{\Delta}$, will be in the same class of black hole metrics provided that there exists a mapping of the charges such that

$$
\tilde{\alpha}=\frac{\gamma}{\sqrt{\Delta}}, \quad \tilde{\beta}=\beta, \quad \tilde{\gamma}=\alpha \sqrt{\Delta}, \quad \tilde{\Delta}=\Delta .
$$

$$
r \rightarrow \tilde{r}=\frac{\sqrt{\Delta}}{r},
$$


[1] W. E. Couch and R. J. Torrence, Conformal invariance under spatial inversion of extreme Riessner-Nordström black holes, Gen. Relativ. Gravit. 16, 789 (1984).

[2] P. Bizon and H. Friedrich, A remark about wave equations on the extreme Reissner-Nordström black hole exterior, Classical Quantum Gravity 30, 065001 (2013).

[3] J. Lucietti, K. Murata, H. S. Reall, and N. Tanahashi, On the horizon instability of an extreme Reissner-Nordström black hole, J. High Energy Phys. 03 (2013) 035.

[4] H. Godazgar, M. Godazgar, and C. N. Pope, Aretakis charges and asymptotic null infinity, Phys. Rev. D 96, 084055 (2017).

[5] S. Aretakis, Stability and instability of extreme ReissnerNordström black hole spacetimes for linear scalar perturbations I, Commun. Math. Phys. 307, 17 (2011).

[6] S. Aretakis, Stability and instability of extreme ReissnerNordström black hole spacetimes for linear scalar perturbations II, Ann. Henri Poincare 12, 1491 (2011).

[7] M. Cvetič and D. Youm, Dyonic BPS saturated black holes of heterotic string on a six torus, Phys. Rev. D 53, R584 (1996).

[8] M. Cvetič and D.Youm, Entropy of nonextreme charged rotating black holes in string theory, Phys. Rev. D 54, 2612 (1996).

[9] Z. W. Chong, M. Cvetič, H. Lü, and C. N. Pope, Charged rotating black holes in four-dimensional gauged and ungauged supergravities, Nucl. Phys. B717, 246 (2005).

[10] M. Cvetič and F. Larsen, Grey body factors for rotating black holes in four-dimensions, Nucl. Phys. B506, 107 (1997).
[11] D. D. K. Chow and G. Compère, Black holes in $N=8$ supergravity from $S O(4,4)$ hidden symmetries, Phys. Rev. D 90, 025029 (2014).

[12] M. Cvetič and A. A. Tseytlin, Solitonic strings and BPS saturated dyonic black holes, Phys. Rev. D 53, 5619 (1996).

[13] E. G. Gimon, F. Larsen, and J. Simon, Black holes in supergravity: The non-BPS branch, J. High Energy Phys. 01 (2008) 040.

[14] M. Cvetič and A. Satz, General relation between Aretakis charge and Newman-Penrose charge, Phys. Rev. D 98, 124035 (2018).

[15] M. Cvetič, C. Pope, A. Saha, and A. Satz, Gaussian null coordinates for rotating charged black holes and conserved charges, Phys. Rev. D 101, 066025 (2020).

[16] M. Cvetič, P. J. Porfirio, and A. Satz, Gaussian null coordinates, near-horizon geometry and conserved charges on the horizon of extremal non-dilatonic black $p$-branes, arXiv:2003.09304.

[17] M. Cvetič, C. N. Pope, and A. Saha, UPR-1306-T (to be published).

[18] L. Borsten, M. J. Duf, and A. Marrani, Freudenthal duality and conformal isometries of extremal black holes, arXiv:1812.10076.

[19] M. Cvetič, M. J. Duff, P. Hoxha, J. T. Liu, H. Lü, J. Lu, R. Martinez-Acosta, C. N. Pope, H. Sati, and T. A. Tran, Embedding AdS black holes in ten-dimensions and eleven-dimensions, Nucl. Phys. B558, 96 (1999).

[20] D.Z. Freedman and A. Van Proeyen, Supergravity (Cambridge University Press, Cambridge, England, 2012). 